\title{
QuizArt: Um aplicativo para auxiliar o Ensino e a Aprendizagem de Arte
}

\author{
Leandro Viana Martins ${ }^{1}$, Anderson Corrêa de Lima ${ }^{1}$, Eseteic Janaina Santos Batista ${ }^{2}$ \\ ${ }^{1}$ Universidade Federal de Mato Grosso do Sul - Campus Ponta Porã \\ ${ }^{2}$ Instituto Federal de Mato Grosso do Sul - Campus Ponta Porã \\ \{leaxviana140, anderson.correa.lima, esteicejanaina\}@gmail.com
}

\begin{abstract}
This article presents a prototype of an application for mobile devices (Android and iOS) whose purpose is to assist in the teaching and learning process of the Classroom Art discipline. Our target audience consists of teachers and students from schools who contemplate teaching art as a subject in their curriculum's. We believe that the application can assist the teacher in teaching art topics by means of a registration form and permission to edit content and a quiz game. In our literature search, we did not find the description of educational applications similar to QuizArt in Portuguese, a fact that underscores the importance of this work.
\end{abstract}

Resumo. Este trabalho apresenta um protótipo de um aplicativo para dispositivos móveis (Android e iOS) cujo objetivo é auxiliar no processo de ensino e aprendizagem da disciplina de Arte em sala de aula. Nosso público-alvo é composto por professores e alunos de escolas que comtemplem o ensino de arte como uma disciplina em sua matriz curricular. Consideramos que o aplicativo pode auxiliar o professor no ensino de tópicos de arte por meio de cadastro de contéudo e pela permissão para edição de um jogo do tipo quiz. Em nossa pesquisa na literatura não encontramos a descrição de aplicativos educacionais similares ao QuizArt em português, fato este que ressalta a importância deste trabalho.

\section{Cenário de Uso}

As formas de aprender e ensinar estão em constante modificação, principalmente nesta última década com o advento da internet das coisas. Em muitas instituições o professor tem deixado de ser o único detentor do conhecimento no ensino e tem passado a assumir o papel de mediador ou de auxiliar o aluno no processo da busca pelo conhecimento. A TDIC (Tecnologia Digital da Informação e Comunicação) é uma das responsáveis por uma contribuição direta nesse novo conceito de apreder e estudar [Pozo 2004]. Recentemente, passamos a utilizar a computação móvel aplicada na educação, o que deu origem a Educação Móvel (Mobile Learnig), que diz respeito ao acesso à informações e ao conteúdo para aprendizagem em qualquer hora e lugar, o que tem favorecido a autonomia do usuário em seu aprendizado.

Atualmente, tratando-se de novas tecnologias aplicadas a educação podemos destacar os Objetos de Aprendizagem (Learning Objects), que correspondem a materiais pedagógicos de ensino elaborados com o uso recursos tecnológicos e reutilizáveis. Estes 
VII Congresso Brasileiro de Informática na Educação (CBIE 2018)

Anais dos Workshops do VII Congresso Brasileiro de Informática na Educação (WCBIE 2018)

objetos podem ser utilizados de uma forma individual, como ferramentas para auxiliar na fixação de um determinado conteúdo, ou de forma coletiva, aplicada a uma turma de alunos com base no uso de aplicativos e jogos educacionais para dispositivos móveis como tablets e smartphones, pois estes apresentam uma forma interessante e atrativa de se obter conhecimentos [Alexandre and Sabbatini 2013]. Com isso, temos que as tecnologias utilizadas em sala de aula podem suplementar e apoiar o processo de aprendizagem [Kesseler 2010].

Particularmente, para o ensino da disciplina de arte, os aplicativos existentes são em sua maioria editores de imagens ou são voltados apenas para o ensino de música. Em nossa pesquisa pela literatura ou nas lojas virtuais, não encontramos aplicativos educacionais que fossem capazes de propocionar a edição de conteúdo, por parte do instrutor e a posterior criação de uma avaliação. Na pesquisa que efetuamos encontramos apenas alguns trabalhos que utilizaram aplicativos já prontos para auxiliar nas disciplinas de arte em sala de aula no Brasil. Em uma delas os autores utilizaram um aplicativo denominado Estúdio Stop Motion como recurso utilizável em dispositivos móveis na criação de vídeos com a técnica de Stop Motion. O stop motion é uma técnica cinematográfica simples e de fácil aprendizado, de custo baixo e não exige muito conhecimento técnico. O aplicativo está disponível para download nas principais lojas virtuais [de Freitas et al. 2016]. Em um outro trabalho foi realizada a imersão dos estudantes em processos artísticos complexos, como a projeção de uma cena ou a recriação de um personagem, isto permitiu que eles valorizassem as manifestações artísticas. Neste caso os autores utilizaram dispositivos tecnológicos como tablets e softwares para edição de fotos e criação de infográficos [Rapkewicz et al. 2014].

A arte, em sua síntese, é a representatividade de expressar ou implementar algum ato de acordo com certos métodos. Ela permite a admissão de saberes para a elaboração de uma criação, de um produto ou de uma concepção, seja por meio da dança, música, cinema e teatro, fotografia, escultura, pintura ou literatura [Lima 2010]. A arte promove o desenvolvimento persuasivo, da idealização da poética pessoal e promove também o desenvolvimento da capacidade imaginativa [Coleto 2010].

Dada a importância da disciplina de arte, este trabalho tem por objetivo apresentar uma ferramenta denominada QuizArt, que consideramos ser capaz de auxiliar no processo de ensino e apredizagem nos diversos conteúdos dos ramos da arte. Nós consideramos que o aplicativo é bastante relevante, visto que educadores e críticos de arte tem buscado experimentações conscientes e atuais das abordagens da arte [Barbosa 2001].

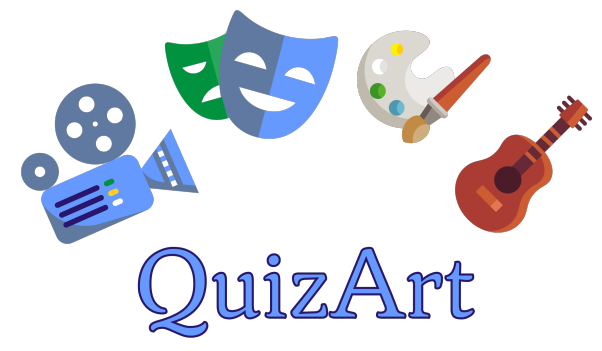

Figura 1. Logo do Aplicativo QuizArt.

O escopo do aplicativo esta vinculado com algumas das trilhas da arte. A arte, como um todo, aborda as seguintes disciplinas: Cinema; Dança; Escultura; Fotografia; 
VII Congresso Brasileiro de Informática na Educação (CBIE 2018)

Anais dos Workshops do VII Congresso Brasileiro de Informática na Educação (WCBIE 2018)

Literatura; Pintura e Teatro. No QuizArt existe a divisão das trilhas em submenus. Dada a quantidade de opções, para facilitar a interação com o usuário, optamos por manter duas trilhas em cada um dos submenus, evitando assim a dispersão do usário pela interface. Muitas opções, cliques e botões podem confundir o usuário [Rogers et al. 2013]. A categorização em submenus do QuizArt é apresentado pela Figura 2.
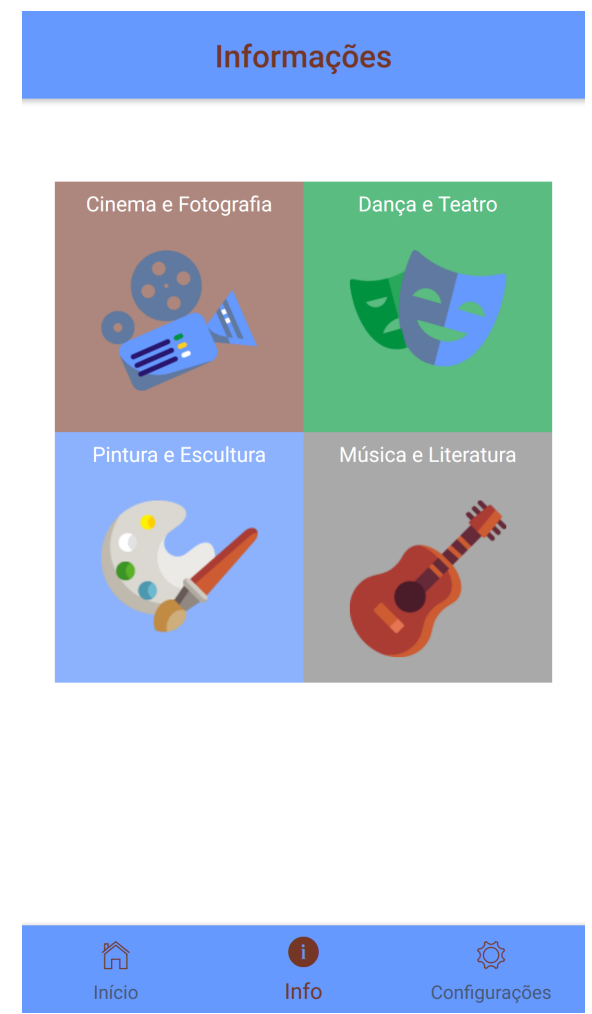

Figura 2. Apresentação ao usuário dos submenus do QuizArt

\section{Desenvolvimento}

A metodologia adotada para o desenvolvimento do aplicativo é a FDD (Feature Driven Development), em português, Desenvolvimento Guiado por Funcionalidades. A FDD é uma metodologia de desenvolvimento de software que inclui alguns benefícios de processos rigorosos, como modelagem, planejamento prévio e controle do projeto, assim como contém características de processos ágeis, como foco na programação, interação constante com o cliente e entrega frequente de versão do produto. Prevê práticas apenas para o desenvolvimento de software em si não se preocupando com outros fatores como a escolha de tecnologias e ferramentas, a definição de procedimentos de aquisição, dentre outros [Silva et al. 2009, Palmer and Felsing 2001].

A FDD faz parte das metodologias ágeis para desenvolvimento de software, que incluem quatro valores principais: interações e indivíduos são mais importantes que processos e ferramentas; o software funcionando é mais relevante que a documentação compreensiva; o cliente deve estar sempre presente durante o projeto e a metodologia deve prover agilidade na resposta a mudanças [Silva et al. 2009].

São cinco os processos da metodologia ágil FDD: Desenvolver um Modelo Abrangente, Construir uma Lista de Funcionalidades, Planejar Através de Funcio- 
VII Congresso Brasileiro de Informática na Educação (CBIE 2018)

Anais dos Workshops do VII Congresso Brasileiro de Informática na Educação (WCBIE 2018)

nalidades, Projetar Através de Funcionalidades e Construir Através de Funcionalidades [Silva et al. 2009]. Uma vez que a FDD não é voltada para produção de vasta documentação, em se tratando de projeto lógico do aplicativo QuizArt optou-se por determinar o encadeamento das funcionalidades que já foram e as que ainda serão desenvolvidas, por meio de diagramas, assimo como descrito no trabalho de Hunt e John [Hunt 2006]. As Figuras 3 e 4 apresentam exemplos dos diagramas que foram produzidos para o QuizArt utilizando a abordagem de diagramas [Hunt 2006].

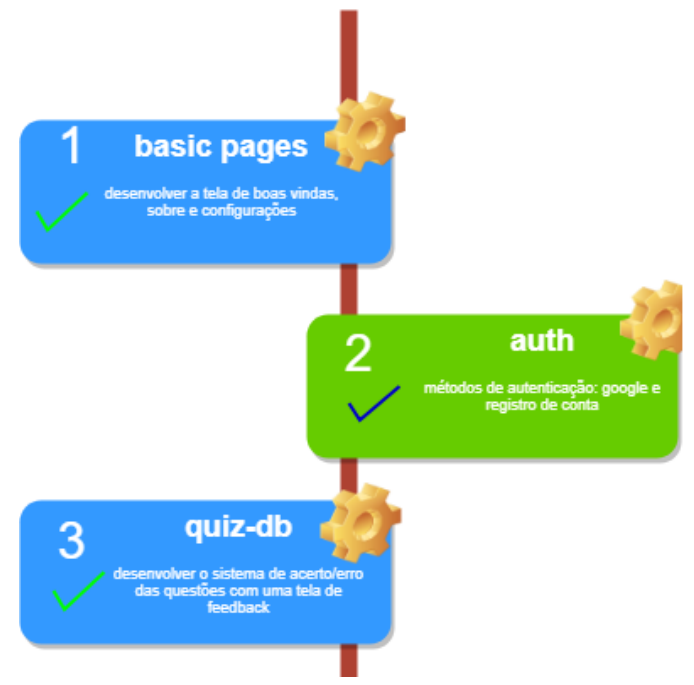

Figura 3. Diagrama 1

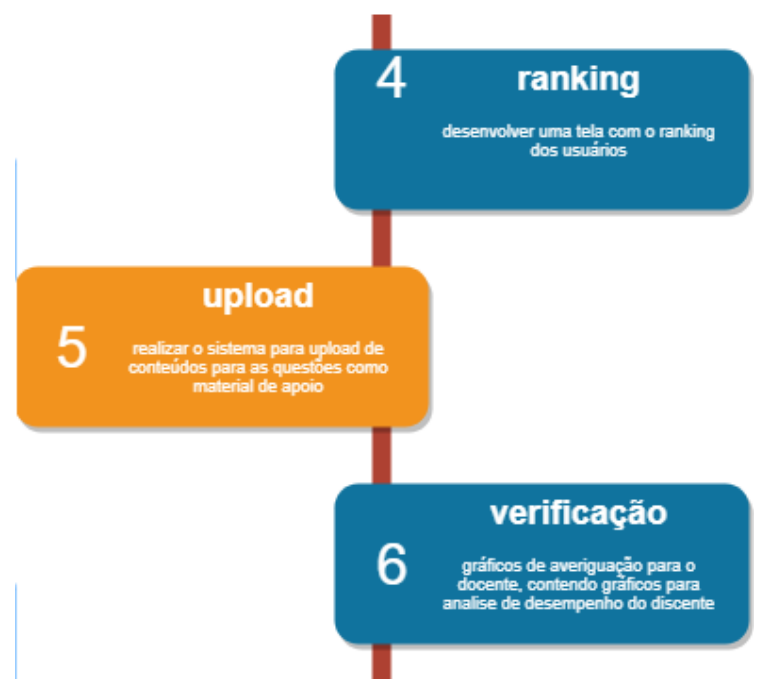

Figura 4. Diagrama 2

Para o desenvolvimento e codificação do aplicativo optou-se pelo framework Ionic. Trata-se de uma ferramenta open-source para desenvolvimento de aplicativos híbridos, isto é, aplicativos com uma única base de código para múltiplas plataformas. Para tal, a ferramenta recorre à tecnologias de desenvolvimento web, tais como: CSS, HTML e Typescript. Com o Typescript podemos desenvolver tanto as demandas do frontend, quanto aquelas do back-end de uma aplicação. A Figura 5 apresenta o ambiente de emulação do Ionic para o aplicativo QuizArt.

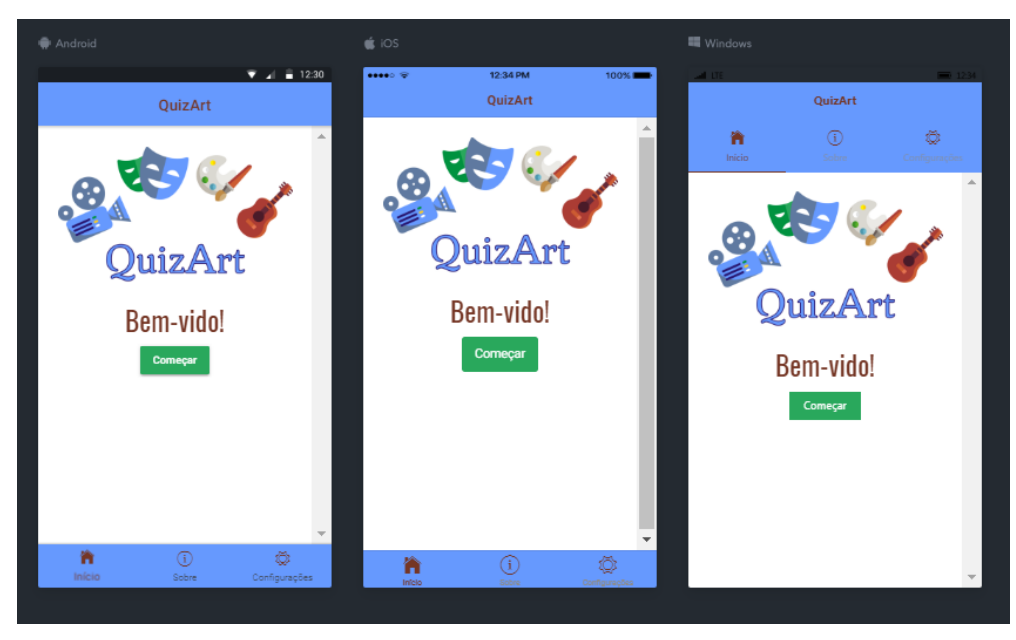

Figura 5. Emulação do QuizArt lonic 
VII Congresso Brasileiro de Informática na Educação (CBIE 2018)

Anais dos Workshops do VII Congresso Brasileiro de Informática na Educação (WCBIE 2018)

Em relação à base dados optamos pelo Firebase, que é um "Back-end as a Service”) (BaaS) adquirido pelo Google ${ }^{\mathrm{TM}}$. Trata-se de uma plataforma de desenvolvimento para aplicações web e mobile [Lardinois 2014]. A plataforma é um banco de dados não relacional e NoSQL, ou seja, não possui linguagem de consulta estruturada. Consideramos que com a utilização dessa plataforma é possível acelerar o desempenho na base de dados, devido ao fato de que seus servidores estão geograficamente localizados no Brasil.

É preciso ressaltar também que durante a fase de testes da codificação das questões do QuizArt nós utilizamos a API Open Trivia Database, especificamente para os testes de feedback de acertos e erros de uma questão. Futuramente, com um banco de dados consolidado, possivelmente na versão final do aplicativo, nós faremos uso de opções mais robustas.

\section{Apresentação do software}

O QuiZart foi projetado para ser executado em todas as plataformas, no entanto, o foco atual está nos ambientes móveis, são eles: Android, iOS e Windows Phone. O design de interação com o aplicatio foi projetado para ser ágil e simples, ele é apresentado a seguir.

Inicialmente o usuário pode escolher entre fazer o login, registrar um e-mail e senha ou cadastrar-se com o Google. Logo depois de efetuada a sua escolha, o utilizador do aplicativo será redirecionado à tela de boas vindas. A autenticação via e-mail e senha será necessária para o desenvolvimento da interface de ranking e do sistema de cadastro de questões. As Figuras 6 e 7 apresentam as telas de login e de boas-vindas do aplicativo.

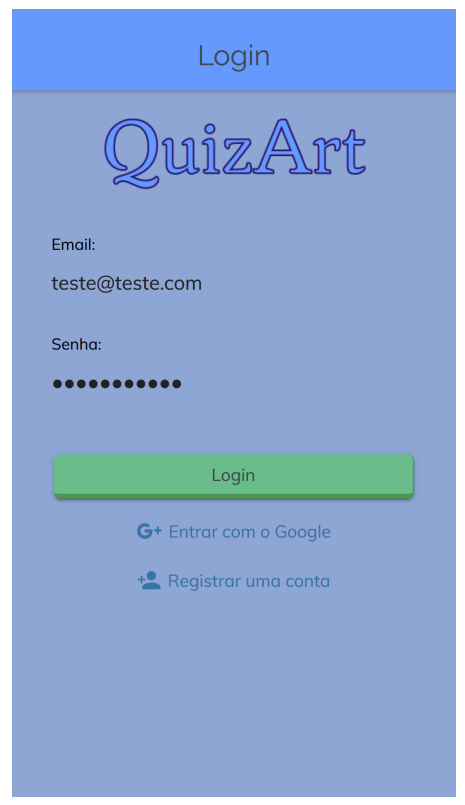

Figura 6. Tela de Login

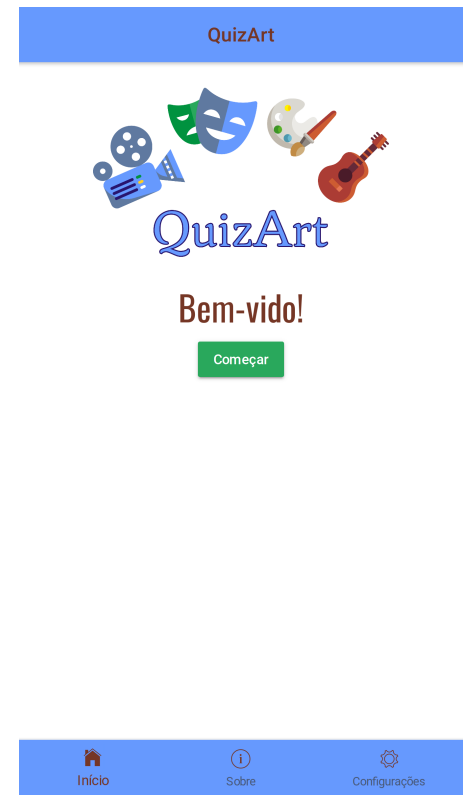

Figura 7. Tela de Boas-Vindas

Uma vez efetuado o login ou o registro, o usuário passa a ter acesso aos tópicos de conteúdo contidos no aplicativo, como já foi apresentado pela Figura 2. Além destas interfaces iniciais, existe uma interface de configurações, na qual o usuário poderá definir o ramo de arte e a dificuldade das questões do Quiz vinculadas a ele. Pode-se escolher um de três níveis: fácil, médio ou difícil. Além disso também será possível escolher o número 
VII Congresso Brasileiro de Informática na Educação (CBIE 2018)

Anais dos Workshops do VII Congresso Brasileiro de Informática na Educação (WCBIE 2018)

de perguntas que o usuário deseja responder no QuizArt. As Figuras 8 e 9 apresentam as interfaces de configurações disponibilizadas até agora.

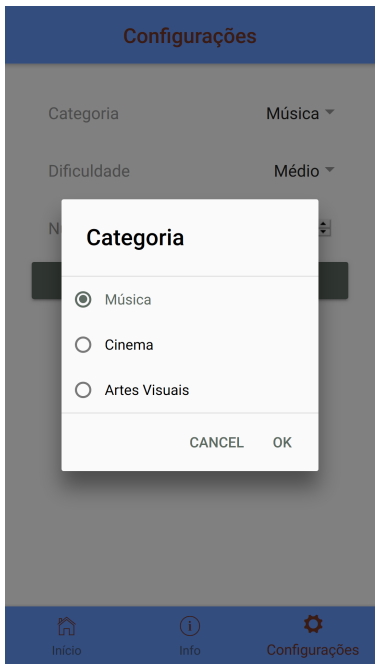

Figura 8. Categorias

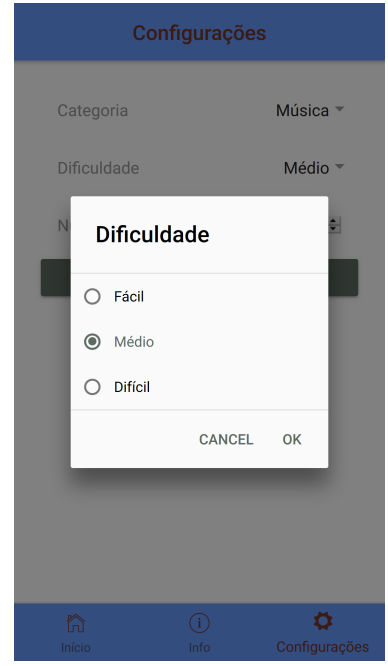

Figura 9. Dificuldade

Na próxima etapa é possível iniciar a resolução das questões clicando no botão "começar"na aba Home, como já visto pela Figura 7. O software obtém as configurações via JSON (JavaScript Object Notation) - uma formatação de troca de informações que ocorre de maneira simples dinâmica - e as usa como parâmetros para a disponibilização das questões no aplicativo. Como trata-se de um software em desenvolvimento, estamos utilizando para testes uma API para o sistema de questões, que já contém diversas perguntas previamente cadastradas. Dessa forma, ainda que na versão protótipo, nós conseguimos analisar os acertos e os erros do usuário-jogador a cada questão fornecendo a ele um feedback sobre seu desempenho. As Figuras 10, 11 e 12 apresentam as interfaces relacionadas a estas funcionalidades.

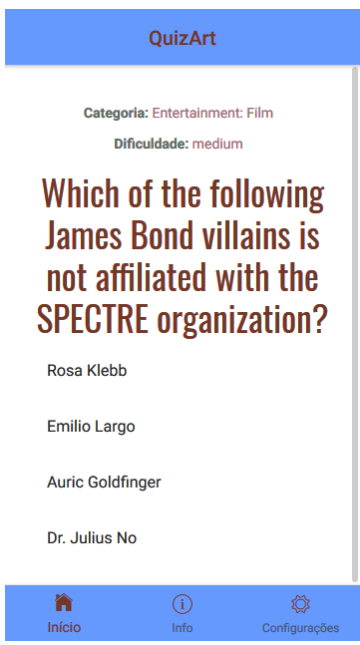

Figura Questão teste

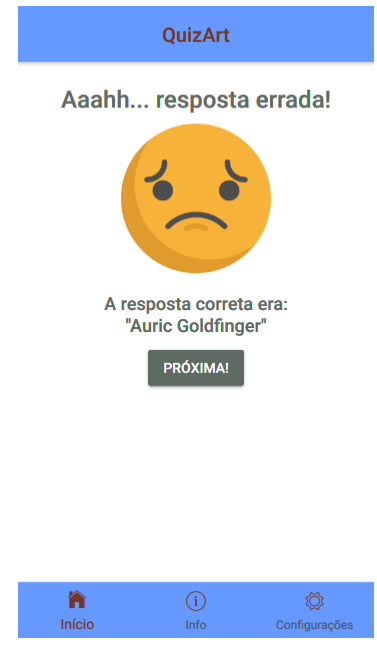

Figura 11. Resposta errada

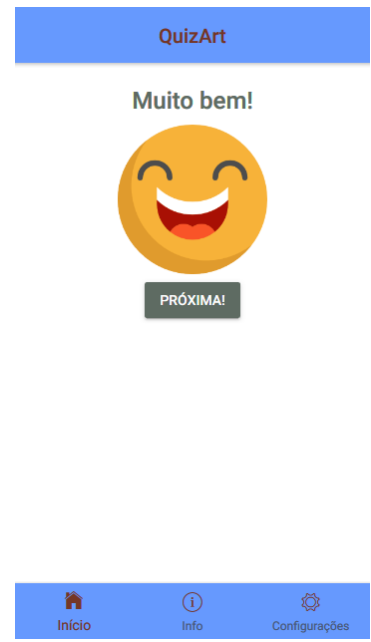

Figura 12. Resposta correta

O QuizArt poderá ser utilizado em sala de aula e possuirá dois perfis: professor e aluno. Em se tratando do perfil do professor, o aplicativo disponibiliza a ele a capacidade 
VII Congresso Brasileiro de Informática na Educação (CBIE 2018)

Anais dos Workshops do VII Congresso Brasileiro de Informática na Educação (WCBIE 2018)

de cadastrar as questões no banco de dados, especificando a trilha, a dificuldade e preenchendo os campos de alternativas. As Figuras 13 e 14 apresentam estas funcionalidades no QuizArt.

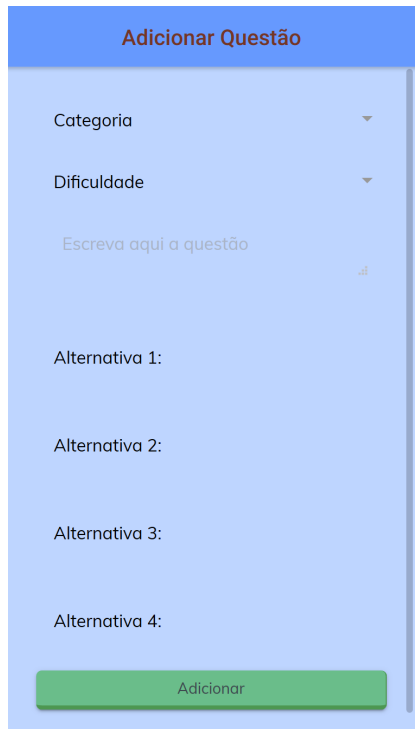

Figura 13. Cadastro de questões

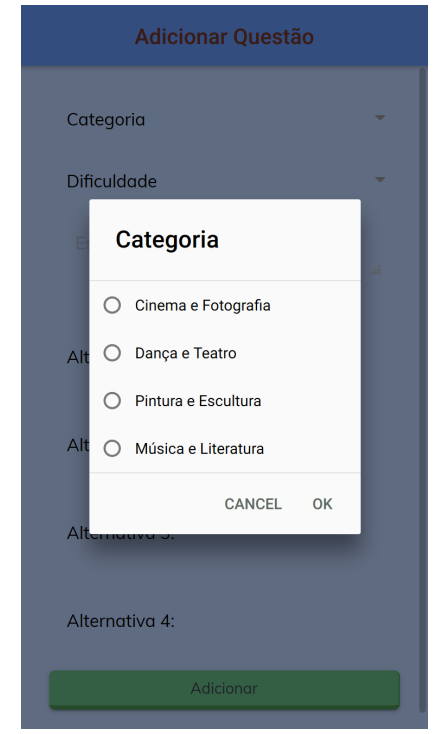

Figura 14. Interface da janela de cadastro

\section{Considerações Finais}

O QuizArt é um aplicativo que foi idealizado neste ano, de 2018, atualmente ele se encontra em fase de desenvolvimento. Para a versão final pretendemos aplicar melhorias no design de interação e nas funcionalidades da aplicação. Consideramos, que uma vez terminado, o aplicativo possa contribuir e auxiliar nas atividades de professores e alunos, em sala de aula, em disciplinas relacionadas aos diversos ramos da arte. Até o momento nosso protótipo já representa grande parte da primeira versão final e funcional do sistema. Para a validação de conteúdo e sugestões de alteração no design do aplicativo, consideramos a consulta a professores das disciplinas de arte, além da consulta na literatura sobre o tema. Por fim, nós consideramos que protótipo do QuizArt já é relevante e atrativo, pois sua proposta é inovadora no que se diz respeito à utilização de TDIC's nas aulas de arte.

Por fim, gostariamos de agradecer o suporte financeiro e estrutural oferecido pela Universidade Federal de Mato Grosso do Sul.

\section{Referências}

Alexandre, C. and Sabbatini, M. (2013). A contribuição dos jogos digitais nos processos de aprendizagem. $5^{\circ}$ Simpósio Hipertexto e Tecnologias na Educação, pages 1-18.

Barbosa, A. M. (2001). John Dewey e o ensino da arte no Brasil, volume 200. São Paulo: Cortez.

Coleto, D. C. (2010). A importância da arte para a formação da criança. Revista Conteúdo, 1(3):137-152. 
VII Congresso Brasileiro de Informática na Educação (CBIE 2018)

Anais dos Workshops do VII Congresso Brasileiro de Informática na Educação (WCBIE 2018)

de Freitas, N. A., Marinho, I. W. T., dos Santos, M. N., and Neto, O. B. (2016). Prática pedagógica com celular e stop-motion em interdisciplinaridade com artes. In Anais do Workshop de Informática na Escola, volume 22, page 953.

Hunt, J. (2006). Feature-driven development. Agile Software Construction, pages 161182.

Kesseler, L. N. (2010). O uso das tic em sala de aula.

Lardinois, F. (2014). Google acquires firebase to help developers build better real-time apps.

Lima, A. (2010). EducaÇÃo E Arte. Clube de Autores.

Palmer, S. R. and Felsing, M. (2001). A practical guide to feature-driven development. Pearson Education.

Pozo, J. I. (2004). A sociedade da aprendizagem e o desafio de converter informação em conhecimento. Revista Pátio, 8(31).

Rapkewicz, C. E., Rodrigues, L., and Bauermann, L. (2014). Manifestações expressivas na inclusão digital: Ações integradas entre as artes e a informática na educação de jovens e adultos. In Anais do Workshop de Informática na Escola, volume 20, page 258.

Rogers, Y., Sharp, H., and Preece, J. (2013). Design de interação: além da interação humano-computador. Bookman.

Silva, F., Hoentsch, S. C., and Silva, L. (2009). Uma análise das metodologias ágeis fdd e scrum sob a perspectiva do modelo de qualidade mps. br. Scientia Plena, 5(12). 